\title{
INTEGRATED COSINE FUNCTIONS
}

\section{QUAN ZHENG}

Dept. of Math., Huazhong Iniv. of Science and Technology, Wuhan 430074, P.R.C.hina

(Received June 18, 1993 and in revised form June 20, 1994)

ABSTRAC:T. In order to the second order Cauchy problem $\left(\mathrm{CP}_{2}\right): x^{\prime \prime}(t)=A x(t), x(0)=x \in$ $D\left(A^{n}\right), x^{\prime \prime}(0)=y \in D\left(A^{m}\right)$ on a Banach space, Arendt and Kellermann recently introduced the integrated cosine function. This paper is concerned with its basic theory, which contain some properties, perturbation and approximation theorems, the relationship to analytic integrated semigroups, interpolation and extrapolation theorems.

KEY WORDS AND PHRASES. Integrated cosine function, analytic integrated semigroup, perturbation, approximation, interpolation, extrapolation.

1991 AMS SUBJECT CLASSIFICATION CODE. 47D09.

\section{BASIC: PROPERTIES}

Let $A$ be a linear operator on a Banach space $X$. If there exist $n \in \mathbf{N}_{0} \equiv \mathrm{N} \cup\{0\}, M, \omega>0$ and a strongly continuous family $C(t)$ in $L(X)$ with $\|C(t)\| \leq M e^{\omega t}$ for $t \geq 0$ such that $\left(\omega^{2}, \infty\right) \subset \rho(A)$ and $R\left(\lambda^{2}, A\right)=\lambda^{n-1} \int_{0}^{\infty} e^{-\lambda t} C(t) d t$ for $\lambda>\omega$, then we say that $A$ generates the (exponentially bounded) $n$-times integrated cosine function $C(t)$ (see [1]), and write $(A, C(t))$ (or $\mathrm{A}$, or $C(t)) \epsilon$ $G_{n}(M, \omega, X)$. Set $G_{n}(\omega, X)=\cup_{M>0} G_{n}(M, \omega, X)$ and $G_{n}(X)=\cup_{\omega>0} G_{n}(\omega, X)$. The definition of integrated semigroups see e.g. [6], and the corresponding notations $\bar{G}_{n}(M, \omega, X), \bar{G}_{n}(\omega, X)$ and $\bar{G}_{n}(X)$ also can be introduced.

It is known that a 0 -times integrated cosine function consists with a cosine function, while the following relationship can be shown by the same method as in [4, Th.3.5].

PROPOSITION 1.1. $(A, C(t)) \in G_{n}(X)$ iff $(\mathcal{A}, \mathcal{C}(t)) \in \bar{G}_{n+1}\left(X^{2}\right)$, where $\mathcal{A}=\left(\begin{array}{ll}0 & I \\ A & 0\end{array}\right)$ and

$$
\mathcal{C}(t)=\left(\begin{array}{cc}
\int_{0}^{t} C(s) d s & \int_{0}^{t}(t-s) C(s) d s \\
C(t)-t^{n} I / n ! & \int_{0}^{t} C(s) d s
\end{array}\right) \text { for } t \geq 0 .
$$

The basic properties of integrated cosine functions now can be deduced from Prop.1.1, the properties of Laplace transforms and integrated semigroups (cf. [6]). We omit the details.

PROPOSITION 1.2. Let $(A, C(t)) \in G_{n}(M, \omega, X),\langle k\rangle=\left[\frac{k+1}{2}\right]$, and $t, s \in \mathbf{R}^{+} \equiv[0, \infty)$. Then

(a) $C(t) C(s)=C(s) C(t), C(r) x=0(r \geq 0)$ implies $x=0$, and $C(t)$ is uniquely determined by $A$.

(b) $\left(A, \int_{0}^{t}(t-s)^{k} C(s) d s / k !\right) \in G_{n+k+1}\left(M \omega^{-k-1}, \omega, X\right)$ for $k \in \mathbf{N}_{0}$.

(c) $R\left(\lambda^{2}, A\right)=\lambda^{n-1} \int_{0}^{\infty} e^{-\lambda t} C(t) d t$ and $\left\|R\left(\lambda^{2}, A\right)\right\| \leq M|\lambda|^{n-1}(\operatorname{Re} \lambda-\omega)^{-1}$ for $\operatorname{Re} \lambda>\omega$.

(d) For $x \in D\left(A^{(k)}\right), C(\cdot) x \in C^{k}\left(R^{+}, X\right)\left(k \in \mathrm{N}_{0}\right)$ and $\lambda^{2-n+k} R\left(\lambda^{2}, A\right) x \rightarrow C^{(k)}(0)=\delta_{k n} x$ as $\lambda \rightarrow \infty(0 \leq k \leq n)$, where $\delta$ denotes the Kronecker delta.

(e) $C(t) x \in \overline{D(A)}, \int_{0}^{t}(t-s) C(s) x d s \in \dot{D}(A)$ and $A \int_{0}^{t}(t-s) C(s) x d s=C(t) x-t^{n} x / n$ ! for $x \in X ; A C(t) x=C(t) A x$ and $C(t) x=\int_{0}^{t}(t-s) C(s) A x d s+t^{n} x / n !$ for $x \in D(A)$.

(f) $\int_{0}^{t} C(s) x d s \in D(A)$ and $A \int_{0}^{t} C(s) x d s=C^{\prime}(t) x-t^{n-1} x /(n-1)$ ! for $x \in X_{1} ; C(t) x \in D(A)$ and $A C(t) x=C^{\prime \prime}(t) x-t^{n-2} x /(n-2)$ ! for $x \in X_{2}$, where $X_{2}=\left\{x \in X ; C(\cdot) x \in C^{\prime}\left(R^{+}, X\right)\right\}$. 
(g) $C(t) \cdot S^{\prime}(s)+S(t) C(s)=\left\{\int_{t}^{t+s}-\int_{0}^{s}\right\}(s+t-r)^{n-1} S(r) d r /(n-1)$ !, where $S(t)=\int_{0}^{t} C(s) d s$.

We conclude this section with some remarks on $\mathrm{CP}_{2} . r(t)$ is called a solution of $\mathrm{CP}_{2}$ if $x(\cdot) \in$ $C^{2}\left(R^{+}, \mathrm{X}\right) \cap C\left(R^{+}, D(A)\right)$ satisfying $\mathrm{CP}_{2}$. CP $\mathrm{P}_{2}$ is called $n$-wellposed if $\mathrm{CP}_{2}$ has a unique solution $x(t)$ for every $(x, y) \in D\left(A^{\langle n+2\rangle}\right) \times D\left(A^{\langle n+1\rangle}\right)$ satisfying $\|x(t)\| \leq p(t)\left(\|x\|_{(n\rangle}+\|y\|_{(n-1)}\right)$ for some locally bounded function $p(t)$. $\mathrm{CP}_{2}$ is called exponentially $n$-wellposed if, in addition, $p(t)=M e^{\omega t}$.

The following result follows from Prop.1.1, [3, Th.2.5] and [6, §7]. The details are omitted.

THEOREM 1.3. Let $\omega>0, n, m \in \mathrm{N}_{0}$ and $\rho(A) \neq \emptyset$. Then the following statements hold.

(a) If $\mathrm{CP}_{2}$ has a unique solution for every $(x, y) \in D\left(A^{\langle n+2\rangle}\right) \times D\left(A^{(m+1)}\right)$ then $\mathrm{CP}_{2}$ is $k$ wellposed where $k=\max (m, n)$. In particular, $\mathrm{CP}_{2}$ has a unique solution for every $(x, y) \in$ $D\left(A^{(n+2)}\right) \times D\left(A^{(n+1)}\right)$ iff $\mathrm{ACP}_{2}$ is $n$-wellposed.

(b) $A \in G_{n}(\omega, X)$ iff $\mathrm{CP}_{2}$ has a unique solution $x(t)$ with $\left\|x^{\prime \prime}(t)\right\|=O\left(e^{\omega t}\right)$ for every $(x, y) \in$ $D\left(A^{\langle n+2\rangle}\right) \times D\left(A^{(n+1\rangle}\right)$. If, in addition, $\overline{D(A)}=X$ then $A \in G_{n}(\omega, X)$ iff $\mathrm{CP}_{2}$ is exponentially $n$-wellposed.

\section{PERTURBATIONS}

We first consider the perturbation problem of $2 m$-times integrated cosine functions. The following is a generalization of the Takenaka-Okazawa theorem (cf. [10, II $]$ ).

THEOREM 2.1. Let $(A, C(t)) \in G_{2 m}(X)$ and $B$ be a linear operator on $X$ satisfying

$\left(b_{1}\right) D(A) \subset D(B), R(B) \subset D\left(A_{1}^{m}\right)$ and $B R\left(\lambda_{0}, A\right) \in L(X)$ for some $\lambda_{0} \in \rho(A)$, where $R(B)$ denotes the range of $B$, and $A_{1}=\left.A\right|_{\overline{D(A)}}$ (the part of $A$ in $\left.\overline{D(A)}\right)$.

$\left(b_{2}\right) l_{\infty} \equiv \lim _{\lambda \rightarrow \infty} l_{\lambda} \leq \infty$, where $l_{\lambda}=\sup \left\{\int_{0}^{\infty} e^{-\lambda t}\left\|B C^{(2 m-1)}(t) x\right\|_{m} d t ; x \in D\left(A^{m+1}\right),\|x\|_{m} \leq\right.$ 1) and $\|x\|_{m}=\|x\|_{m}^{A}=\sum_{k=0}^{m}\left\|A^{k} x\right\|$ for $x \in D\left(A^{m}\right)$.

Then $A_{1}+q B \in G_{2 m}(\overline{D(A)})$ for every $q$ with $|q|<1 / l_{\infty}$.

PROOF. Let $\mathcal{A}=\left(\begin{array}{ll}0 & I \\ A & 0\end{array}\right), \mathcal{B}=\left(\begin{array}{ll}0 & 0 \\ B & 0\end{array}\right)$ and $\mathcal{C}(t)$ be given by (1) with $n=2 m$. Then

$$
R(\lambda, \mathcal{A})=\left(\begin{array}{cc}
\lambda & I \\
A & \lambda
\end{array}\right) R\left(\lambda^{2}, A\right) \text { for } \lambda^{2} \in \rho(A),
$$

and therefore $\left(b_{1}\right)$ implies $\left(b_{1}^{\prime}\right): D(\mathcal{A}) \subset D(\mathcal{B}), R(\mathcal{B}) \subset D\left(\mathcal{A}_{1}^{2 m+1}\right)$ and $\mathcal{B} R\left(\lambda_{0}^{1 / 2}, \mathcal{A}\right) \in L\left(X^{2}\right)$. Since $|(x, y)|_{m} \equiv\|x\|_{m+1}^{A}+\|y\|_{m}^{A}$ is equivalent to $\|(x, y)\|_{2 m+1}^{A}$ on $D\left(\mathcal{A}^{2 m+1}\right)$ we have that

$$
l_{\lambda}^{\prime} \equiv \sup \left\{\int_{0}^{\infty} e^{-\lambda t}\left|\mathcal{B C} \mathcal{C}^{(2 m+1)}(t)(x, y)\right|_{m} d t ;(x, y) \in D\left(\mathcal{A}^{2 m+2}\right),|(x, y)|_{m} \leq 1\right\} \leq \max \left\{l_{\lambda}^{\prime \prime}, l_{\lambda}\right\},
$$

where $l_{\lambda}^{\prime \prime} \equiv \sup \left\{\int_{0}^{\infty} e^{-\lambda t}\left\|B C^{(2 m)}(t) x\right\|_{m} d t ; x \in D\left(A^{m+1}\right),\|x\|_{m+1} \leq 1\right\}$. Noting that $l_{\lambda} \leq l_{\lambda}^{\prime}$ and, by $\left(b_{1}\right), l_{\lambda}^{\prime \prime} \rightarrow 0$ as $\lambda \rightarrow \infty$, one has that $\lim _{\lambda \rightarrow \infty} l_{\lambda}^{\prime}=l_{\infty}$. Combining $\left(b_{1}^{\prime}\right)$, Prop.1.1, [10, II, Th.4.2] with this yields that $\mathcal{A}_{1}+q \mathcal{B} \in \bar{G}_{2 m+1}(\overline{D(\mathcal{A})})$ for $|q|<1 / l_{\infty}$. Let $\mathcal{C}(t)$ generated by $\mathcal{A}_{1}+q \mathcal{B}$ have the form $\left(\begin{array}{cc}S_{1}(t) & * \\ C_{1}(t)-t^{2 m+1} I /(2 m+1) ! & *\end{array}\right)$. Then we can deduce from (1) and (2) that

$$
\lambda^{2 m-1} \int_{0}^{\infty} e^{-\lambda t} C_{1}(t) x d t=R\left(\lambda^{2}, A_{1}+q B\right) x=\lambda^{2 m} \int_{0}^{\infty} e^{-\lambda t} S_{1}(t) x d t \quad \text { for } x \in \overline{D(A)} .
$$

Consequently $C_{1}(t) \in L(\overline{D(A)})$, and so the claim follows.

The subsequent theorem can be shown by [10, II, Th.4.3] and the same method as above.

THEOREM 2.2. Let $(A, C(t)) \in G_{2 m}(X)$ and $B$ be a closed linear operator on $X$ satisfying $\left(b_{3}\right) D(A) \cup \int_{0}^{t} C(s)\left(X^{\prime}\right) d s \subset D(B)$ for $t \geq 0$ and $R(B) \subset D\left(A^{m}\right)$.

$\left(b_{4}\right) B \int_{0} C(s) x d s \in C\left(R^{+},\left[D\left(A^{m}\right)\right]\right)$ for $x \in X$ where $\left[D\left(A^{m}\right)\right]=\left(D\left(A^{m}\right),\|\cdot\|_{m}\right)$, and $l_{\infty} \equiv$ $\lim _{\lambda \rightarrow \infty} l_{\lambda}<\infty$ where $l_{\lambda}=\sup \left\{\int_{0}^{\infty} e^{-\lambda t}\left\|B C^{(2 m-1)}(t) x\right\|_{m} d t ; x \in D\left(A^{m}\right),\|x\|_{m} \leq 1\right\}$.

Then $A+q B \in G_{2 m}(X)$ for every $q$ with $|q|<1 / l_{\infty}$.

In the case $m=0$, since $l_{\infty}=0$ (see $\left[9\right.$, Lemma]) and $\left(b_{3}\right)$ can be replaced by $\int_{0}^{t} C(s)(X) d s \in$ $D(B)$ for $t \geq 0$, Theorem 3.2 (with $m=0$ ) is consistent with [9, Prop.].

We now turn to the perturbation problem of $(2 m+1)$-times integrated cosine functions. 
THEOREM 2.3. Let $(A, C(t)) \in G_{2 m+1}\left(X^{\prime}\right)$ and $B$ be a linear operator on $X$ satisfying $\left(b_{1}\right)$ and $\left\|B C^{\prime}(t) x\right\|_{m} \leq M e^{\omega t}\|x\|$ for $x \in D(A)$ and $t \geq 0$. Then $A+B \in G_{2 m+1}(\overline{D(A)})$.

PROOF. We first note that $\rho(A) \subset \rho\left(A_{1}\right)$ and $R\left(\lambda, A_{1}\right)=R(\lambda, A)$ on $\overline{D(A)}$ for $\lambda \in \rho(A)$. Next, set $C_{1}(t)=\left(\lambda_{0}-A\right)^{m} B C^{(2 m)}(t) R\left(\lambda_{0}, A\right)^{m}$ and $C_{2}(t)=\left(\lambda_{0}-A_{1}\right)^{m} B C(t)$ on $D(A)$ for some $\lambda_{0} \in \rho(A)$. Then, by our assumptions and Prop.1.2(d), $C_{1}(t)(i=1,2)$ can be extended to strongly continuous families in $L(\overline{D(A)})$, and satisfy $B_{1} R\left(\lambda^{2}, A_{1}\right)=\int_{0}^{\infty} e^{-\lambda t} C_{1}(t) d t$ and $B_{2} R\left(\lambda^{2}, A_{1}\right)=$ $\lambda^{2 m} \int_{0}^{\infty} \mathrm{c}^{-\lambda^{\lambda t}}\left(_{2}(t) d t\right.$ for large $\lambda$, where $B_{1}=\left(\lambda_{0}-A_{1}\right)^{m} B R\left(\lambda_{0}, A_{1}\right)^{m}$ and $B_{2}=\left(\lambda_{0}-A_{1}\right)^{m} B$. It follows thus that

$$
C_{3}^{\prime}(t) \equiv C^{\prime}(t)+\left\{C^{(2 m)}(t) R\left(\lambda_{0}, A_{1}\right)^{m}\right\} *\left\{I+C_{1}(t)+\sum_{k=2}^{\infty} C_{1}(t)^{* k}\right\} * C_{2}(t) \text { for } t \geq 0
$$

is also a strongly continuous family in $L(\overline{D(A)})$, where * denotes the convolution. Now, one can directly check that $R\left(\lambda^{2}, A_{1}+B\right)=\lambda^{2 m} \int_{0}^{\infty} e^{-\lambda t} C_{3}(t) d t$ for large $\lambda$.

By a modification of the proof of Theorem 3.3 we can show the following

THEOREM 2.4. Let $(A, C(t)) \in G_{2 m+1}(X)$ and $B$ be a closed linear operator on $X$ satisfying

$\left(b_{5}\right) D(A) \cup C(t)(X) \subset D(B)$ for $t \geq 0$ and $R(B) \subset D\left(A^{m}\right)$.

$\left(b_{6}\right) B C(\cdot) \cdot x \in C\left(R^{+},\left[D\left(A^{m}\right)\right]\right)$ and $\|B C(t) \cdot x\|_{m} \leq M e^{\omega t}\|x\|$ for $x \in X$ and $t \geq 0$.

Then $A+B \in G_{2 m+1}(X)$.

As a generalization of $[1$, Th.5.3] we have

COROLlarY 2.5. Let $A \in G_{n}(X)$, and let $B \in L(X)$ and $B: \overline{D(A)} \rightarrow D\left(A^{\langle n-1\rangle}\right)$ (or $B: D(A) \rightarrow D\left(A^{(n+1)}\right)$ and $B R\left(\lambda_{0}, A\right) \in L\left(X^{\prime}\right)$ for some $\left.\lambda_{0} \in \rho(A)\right)$. Then $A+B \in G_{n}(X)$.

3. $\Lambda$ PPROXIMATIONS

The first approximation theorem is a direct consequence of [10, I, Th.3].

THEOREM 3.1. Let $\left(A_{k}, C_{k}(t)\right) \in G_{n}(M, \omega, X)\left(k \in \mathrm{N}_{0}\right)$. If $\lim _{k \rightarrow \infty} R\left(\lambda_{0}^{2}, A_{k}\right) x=R\left(\lambda_{0}^{2}, A_{0}\right) x$ for $x \in X$ and some $\operatorname{Re} \lambda_{0}>\omega$, then for $x \in \overline{D\left(A_{0}\right)}, y \in X$ and $T>0$

$$
\lim _{k \rightarrow \infty} \sup _{0 \leq t \leq T}\left\{\left\|C_{k}(t) x-C_{0}(t) x\right\|+\left\|\int_{0}^{t}\left(C_{k}(s) y-C_{0}(s) y\right) d s\right\|\right\}=0 .
$$

Conversely, if $\overline{D\left(A_{0}\right)}=X^{\prime}$ and $\lim _{k \rightarrow \infty} C_{k}(t) x=C_{0}(t) x$ for $x \in X$ and $t \geq 0$ then $\lim _{k \rightarrow \infty} R\left(\lambda^{2}, A_{k}\right) x$ $=R\left(\lambda^{2}, A_{0}\right) \cdot r$ for $x \in X$, uniformly for $\lambda$ in compact subsets of $\operatorname{Re} \lambda>\omega$.

TIIEOREM 3.2. Let $\left(A_{k}, C_{k}(t)\right) \in G_{n}(M, \omega, X)(k \in \mathrm{N})$, and $\lim _{k \rightarrow \infty} R\left(\lambda_{0}^{2}, A_{k}\right) x=R_{0} x(x \in$ $X)$ for some $\operatorname{Re} \lambda_{0}>\omega$ and some injective operator $R_{0}$. Then there exists $(A, C(t)) \in G_{n+1}(\omega, X)$ such that $\left(A_{1}, C^{\prime}(t)\right) \in G_{n}(M, \omega, \overline{D(A)}), R_{0}=R\left(\lambda_{0}^{2}, A\right)$ and $(3)\left(A_{0}\right.$ and $C_{0}(t)$ replaced by $A$ and $C(t)$, respectively) holds. If, in addition, $R_{0}$ has dense range then $\left(A, C^{\prime}(t)\right) \in G_{n}(M, \omega, X)$.

PROOF. For every $\left(A_{k}, C_{k}(t)\right) \in G_{n}(M, \omega, X)$ we define $\left(\mathcal{A}_{k}, \mathcal{C}_{k}(t)\right) \in \bar{G}_{n+1}\left(\omega, X^{2}\right)$ as in Prop.1.1. Thus (2) and our assumption implies that

$$
\lim _{k \rightarrow \infty} R\left(\lambda_{0}, \mathcal{A}_{k}\right)(x, y)^{\tau}=\left(\begin{array}{cc}
\lambda_{0} R_{0} & R_{0} \\
-I+\lambda_{0}^{2} R_{0} & \lambda_{0} R_{0}
\end{array}\right)\left(\begin{array}{l}
x \\
y
\end{array}\right) \equiv \mathcal{R}_{0}(x, y)^{\top} \quad \text { for } x, y \in X .
$$

It follows from $[10, \mathrm{I}, \mathrm{Th} .4]$ that there exists $(\mathcal{A}, \mathcal{C}(t)) \in \bar{G}_{n+2}\left(\omega, X^{2}\right)$ such that $\mathcal{R}_{0}=R\left(\lambda_{0}, \mathcal{A}\right)$ and

$$
\lim _{k \rightarrow \infty} \sup _{0 \leq t \leq T}\left\|\int_{0}^{t} \mathcal{C}_{k}(s)(x, y)^{\tau} d s-\mathcal{C}(t)(x, y)^{\top}\right\|=0 \quad \text { for } x, y \in X, T>0
$$

It is easy to show, by $\mathcal{R}_{0}=R\left(\lambda_{0}, \mathcal{A}\right)$, that $\mathcal{A}=\left(\begin{array}{ll}0 & I \\ A & 0\end{array}\right)$ and $R_{0}=R\left(\lambda_{0}^{2}, A\right)$, while the remainder conclusions now can be deduced from Prop.1.1 and (4).

\section{RELA'TIONSHIP TO INTEGRATED SEMIGROUPS}

Let $A$ be a linear operator on $X$. If there exist $n \in \mathrm{N}_{0}, \omega>0$ and a strongly continuous family $T(t) \in L(X)(t>0)$ such that $(\omega, \infty) \subset \rho(A), \int_{0}^{\infty} e^{-\lambda t}\|T(i)\| d t<\infty$ and $R(\lambda, A)=$ $\lambda^{n} \int_{0}^{\infty} e^{-\lambda t} T(t) d t$ for $\lambda>\omega$, then we say that $A$ generates the $n$-times semigroup $T(t)$, and write 
$(\Lambda, T(t)) \in \tilde{G}_{n}\left(X^{\prime}\right)$. Obviously, $\left(A, S^{\prime}(t)\right) \in \bar{G}_{n}\left(X^{\prime}\right)$ implies $(A, S(t)) \in \tilde{G}_{n}\left(X^{\prime}\right)$, and $(A, T(t)) \in$ $\tilde{G}_{n}\left(X^{\prime}\right)$ implies $\left(A, \int_{0}^{t} T(s) d s\right) \in \bar{G}_{n+1}(X)$ (see [2, Prop.1.1]).

LEMMA 4.1. Let $(A, C(t)) \in G_{n}(X)$ and $\Sigma_{\theta}=\{t ;|\arg t|<\theta\} \backslash\{0\}(0<\theta \leq \pi / 2)$. Define

$$
T_{k}(t)= \begin{cases}(-1)^{k}(\pi t)^{-1 / 2} \int_{0}^{\infty}\left[D_{s}^{k} \exp \left(-s^{2} / 4 t\right)\right] C(s) d s & \text { for } k \in \mathbf{N}_{0} \\ (\pi t)^{-1 / 2} \int_{0}^{\infty} \exp \left(-s^{2} / 4 t\right)\left(\int_{0}^{s} C(r) d r\right) d s & \text { for } k=-1,\end{cases}
$$

where $D_{s}=\partial / \partial s$. Then $T_{k}(t)$ is analytic and $T_{k}^{\prime}(t)=T_{k+2}(t)$ for $\operatorname{Re} t>0$ and $k \in \mathbf{N}_{0} \cup\{-1\}$. If in addition $\|C(t)\| \leq M t^{d} e^{\omega t}(t \geq 0)$ for some $d \geq 0$, then for $k \in \mathrm{N}_{0}$ and $0<\theta<\pi / 2$

(a) $\left\|T_{k}(t)\right\| \leq M|t|^{d+1 / 2}(\operatorname{Re} t)^{-(k+d+1) / 2}\left(1+|t|^{2} / \operatorname{Re} t\right)^{(k+d) / 2} \exp \left(\omega^{2}|t|^{2} / \operatorname{Re} t\right)$ for Ret $>0$.

(b) $\left\|T_{k}(t)\right\| \leq M_{\theta}|t|^{(d-k) / 2}(1+|t|)^{(k+d) / 2} \exp \left(\omega^{2} \operatorname{Re} t / \cos ^{2} \theta\right)$ for $t \in \bar{\Sigma}_{\theta} \backslash\{0\}$.

(c) $\left\|T_{-1}(t)\right\| \leq M|t|^{d+3 / 2}(\operatorname{Re} t)^{-(d+2) / 2}\left(1+|t|^{2} / \operatorname{Re} t\right)^{(d+1) / 2} \exp \left(\omega^{2}|t|^{2} / \operatorname{Re} t\right)$ for Ret $>0$.

(d) $\left\|T_{-1}(t)\right\| \leq M_{\theta}|t|^{(d+1) / 2}(1+|t|)^{(d+1) / 2} \exp \left(\omega^{2} \operatorname{Re} t / \cos ^{2} \theta\right)$ for $t \in \bar{\Sigma}_{\theta} \backslash\{0\}$.

(e) In the case $-1 \leq k \leq d+2$ we have that $\int_{0}^{\infty} e^{-\lambda t}\left\|T_{k}(t)\right\| d t<\infty$ and

$$
R(\lambda, A)=\lambda^{(n-k) / 2} \int_{0}^{\infty} e^{-\lambda t} T_{k}(t) d t \text { for } \lambda>\omega^{2} .
$$

PROOF. The analyticity of $T_{k}(t)$ and $T_{k}^{\prime}(t)=T_{k+2}(t)$ are easily shown. By (5) we have

$$
\begin{aligned}
\left\|T_{k}(t)\right\| & \leq M|t|^{-1 / 2} \int_{0}^{\infty} \sum_{i=0}^{\langle k-1\rangle} s^{k-2 t}|t|^{i-k} \exp \left(-s^{2} \operatorname{Re} t / 4|t|^{2}\right) s^{d} e^{\omega s} d s \\
& \leq M \sum_{i=0}^{(k-1\rangle}|t|^{d+\imath+1 / 2}(\operatorname{Re} t)^{i-(k+d+1) / 2} \int_{0}^{\infty} v^{k-2 t+d} \exp \left(-v^{2}-2 \omega|t| v / \sqrt{\operatorname{Re} t}\right) d v \\
& \leq M|t|^{d+1 / 2}(\operatorname{Re} t)^{-(k+d+1) / 2}\left(1+|t|^{2} / \operatorname{Re} t\right)^{(k+d) / 2} \exp \left(\omega^{2}|t|^{2} / \operatorname{Re} t\right)
\end{aligned}
$$

for Ret $>0$ and $k \in \mathrm{N}_{0}$, where $M$ denotes a generic constant. Thus (a) is proved. (b) follows from (a) and Ret $\geq|t| \cos \theta\left(t \in \bar{\Sigma}_{\theta}\right)$. Since $\|C(t)\| \leq M t^{d} e^{\omega t}$ implies $\left\|\int_{0}^{t} C(s) d s\right\| \leq M t^{d+1} e^{\omega t}$, (c) and (d) follow from (a) and (b) (replacing $(k, d)$ with $(0, d+1)$ ), respectively. Finally, $\int_{0}^{\infty} e^{-\lambda t}\left\|T_{k}(t)\right\| d t$ $<\infty$ follows from (b) and (d), while by $T_{k}^{\prime}(t)=T_{k+2}(t)$ and Fubini's theorem we can check (6).

COROLLARY 4.2. Let $(A, C(t)) \in G_{n}(X)$ and $\|C(t)\| \leq M t^{d} e^{\omega t}(t \geq 0)$ for some $0 \leq d \leq n$. If $k=n-2 m$ and $m$ is the least integer $>(n-d-2) / 2$, then $\left(A, T_{k}(t)\right) \in \tilde{G}_{m}(X)$.

In the sequel, we infer to, e.g., $[10, \mathrm{III}]$ for analytic integrated semigroups, and write $(A, S(t)) \in$ $H_{n}(\theta, \omega, X)$ for $A$ generating the analytic $n$-times integrated semigroup $S(t)$ of type $(\theta, \omega)$.

THEOREM 4.3. Let the conditions of Corollary 5.3 be satisfied. Then for every $\theta \in(0, \pi / 2)$

(a) If $k<d$ then $\left(A, S_{k}(t)\right) \in H_{m}\left(\theta, \omega^{2} / \cos ^{2} \theta, X\right)$, where $S_{k}(t)=T_{k}(t)$ for $t \in \Sigma_{\theta}$ and $S_{k}(0)=0$.

(b) In the case $k=d$, if in addition (i) $\overline{D(A)}=X$, or (ii) $\lim _{t \rightarrow 0} C(t) x / t^{k}(x \in D$ ) exist where $\bar{D}=X$, then $\left(A, S_{k}(t)\right) \in H_{m}\left(\theta, \omega^{2} / \cos ^{2} \theta, X\right)$, where $S_{k}(t)=T_{k}(t)$ for $t \in \Sigma_{\theta}$ and $S_{k}(0)=\delta_{k n} I$.

PROOF. (a) follows from Lemma 4.1. To prove (b), we note, by Prop.1.2, that (i) implies (ii)

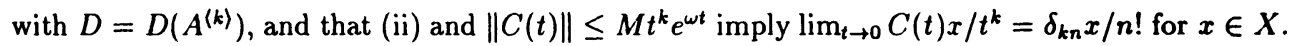
The following proof is divided into cases.

Case $k<n$. Fix $x \in X$. Then for arbitrary $\varepsilon>0$, there exists $\delta>0$ such that $\left\|C(s) x / s^{k}\right\|<\varepsilon$ $(0<s<\delta)$. Thus we have (cf. the proof of Lemma 4.1(a))

$$
\begin{aligned}
\left\|T_{k}(t) x\right\| \leq & M|t|^{-1 / 2} \sum_{t=0}^{(k-1)} \int_{0}^{\delta} s^{k-2 i}|t|^{\mid-k} \exp \left(-s^{2} \operatorname{Re} t / 4|t|^{2}\right) \varepsilon s^{k} d s \\
& +M|t|^{-1 / 2} \sum_{i=0}^{(k-1)} \int_{\delta}^{\infty} s^{k-2 \imath}|t|^{i-k} \exp \left(-s^{2} \operatorname{Re} t / 4|t|^{2}\right) s^{k} e^{\omega s} d s \\
\leq & M_{\theta} \varepsilon+M_{\theta} \exp \left(\omega^{2}|t| / \cos \theta\right) \sum_{t=0}^{(k-1)} \int_{\gamma(t)}^{\infty}\left(u^{2 k-2 \imath}+|t|^{k-1}\right) \exp \left(-u^{2}\right) d u
\end{aligned}
$$

where $\gamma(t)=\delta \sqrt{\operatorname{Re} t} / 2|t|-\omega|t| / \sqrt{\operatorname{Re} t} \rightarrow \infty$ as $\Sigma_{\theta} \ni t \rightarrow 0$. Thus $T_{k}(t) x \rightarrow 0$ as $\Sigma_{\theta} \ni t \rightarrow 0$, i.e., $S_{k}(t)$ is strongly continuous on $\Sigma_{\theta} \cup\{0\}$ and therefore the claim follows from Lemma 4.1.

Case $k=n$. Since $\lim _{t \rightarrow 0} C(t) x / t^{n}=x / n$ ! for $x \in X$, we have (cf. (7))

$$
T_{n}(t) x-x=(-1)^{n}(\pi t)^{-1 / 2} \int_{0}^{\infty}\left[D_{s}^{n} \exp \left(-s^{2} / 4 t\right)\right] s^{n}\left(C(s) x / s^{n}-x / n !\right) d s \rightarrow 0
$$


as $\Sigma_{\theta} \ni t \rightarrow 0$ for $r \in X$ and therefore the claim also follows from Lemma 4.1.

In the case $n=1$, Cor.4.2 (with $d=0$ ) and Th.4.3 (with $d=1$ ) were shown in [1, Th.5.2]. In the casc $d=0$ we obtain

COROLLARY 4.4. Let $(A, C(t)) \in G_{n}(X)$. Then for every $\theta \in(0, \pi / 2)$

(a) If $n=2 m$ then $(A, S(t)) \in H_{m}\left(\theta, \omega^{2} / \cos ^{2} \theta, X\right)$, where $S(t)=T_{0}(t)$ for $t \in \Sigma_{\theta}$ and $S(0)=\delta_{0 m} I$.

(b) If $n=2 m+1$ then $\left(A, T_{1}(t)\right) \in \tilde{G}(X)$ and $(A, S(t)) \in H_{m+1}\left(\theta, \omega^{2} / \cos ^{2} \theta, X\right)$, where $S(t)=T_{-1}(t)$ for $t \in \Sigma_{\theta}$ and $S(0)=0$.

The results relating to Cor.4.4 see $[5,8]$.

\section{INTERPOLATION AND EXTRAPOLATION}

In this section we will give some results on interpolation and extrapolation of integrated cosine functions, which are analogous to the results of integrated semigroups obtained by Arendt et al [2]. In the sequel, $X, Y$ and $Z$ are Banach spaces. $X \hookrightarrow Y$ means that $X$ is a subspace of $Y$ and that the inclusion is continuous. We write $X \hookrightarrow_{d} Y$, if in addition $X$ is dense in $Y$.

THEOREM 5.1. Let $B \in\left(i_{0}(Y)\right.$ and $\left[D\left(A^{m}\right)\right] \hookrightarrow X \hookrightarrow Y$ for some $m \in \mathbf{N}$. In the case $m \geq 2$ assume in addition that $R\left(\lambda_{0}, B\right) X \subset X$ for some $\lambda_{0} \in \rho(B)$. Then $B_{X} \in G_{2 m}(X)$.

PROOF. Let $(B, S(t)) \in G_{0}(\omega, Y)$ and $C(t)=\int_{0}^{t}(t-s)^{2 m-1} S(s) d s /(2 m-1) !$. Since by Prop.1.2(f) (with $n=0)$

${ }^{\prime} C(t)=\sum_{i=0}^{m}\left(\begin{array}{c}m \\ i\end{array}\right) \lambda_{0}^{m-\imath}(-1)^{2}\left\{R\left(\lambda_{0}, B\right)^{m} \int_{0}^{t} \frac{(t-s)^{2 m-2 \imath-1}}{(2 m-2 i-1) !} S(s) d s+\sum_{\jmath=m-\imath}^{m-1} \frac{t^{2 \jmath}}{(2 j) !} B^{\jmath-m+i} R\left(\lambda_{0}, B\right)^{m}\right\}$

for $t \geq 0$, our assumptions (noting $R\left(\lambda_{0}, B\right) X \subset R\left(\lambda_{0}, B\right) Y \subset D(B) \subset X$ for $m=1$ ) imply the strongly continuity of $C(t)_{X}$ and $\left\|C(t)_{X}\right\|_{X} \leq M e^{\omega t}$. The claim now follows from

$$
\lambda R\left(\lambda^{2}, B_{X}\right)=\lambda R\left(\lambda^{2}, B\right)_{X}=\int_{0}^{\infty} e^{-\lambda t} S(s)_{X} d s=\lambda^{2 m} \int_{0}^{\infty} e^{-\lambda t} C(s)_{X} d s \quad \text { for } \lambda>\omega .
$$

Similarly to [1, Lemma 2.4] we can show that if $(A, C(t)) \in G_{2 m}(Y)$, and if $D(A) \neq Y$ in the case $m=0$ and $C(\cdot) y \notin C\left(R^{+},[D(A)]\right)$ for some $y \in Y$ in the case $m>0$, then $[D(A)] \hookrightarrow X \hookrightarrow Y$ for some $X,\left(A_{X}, S(t)\right) \in G_{2 m+2}(X)$, and $S(\cdot) x \notin C\left(R^{+},\left[D\left(A_{X}\right)\right]\right)$ for some $x \in X$. From this we can deduce the following theorem (cf. the proof of $[1$, Th.0.1(b)]).

THEOREM 5.2. Let $B \in G_{0}(Y)$ and $D(B) \neq Y$. Then $\left[D\left(B^{m}\right)\right] \hookrightarrow X \hookrightarrow Y$ for some $X$, and $B_{X} \in G_{2 m}(X)$.

The converse of Th.5.1 and 5.2, i.e., an extrapolation of integrated cosine functions is as follows.

THEOREM 5.3. Let $A \in G_{2 m}(\omega, X)$. Then $\left[D\left(A^{m}\right)\right] \hookrightarrow X \hookrightarrow_{d} Y$ for some $Y, A=B_{X}$ for some $B \in G_{0}(Y)$, and $\left[D\left(A^{m}\right)\right]$ is maximal unique (i.e., $Z \hookrightarrow X$ and $A_{Z} \in G_{0}(\omega, Z)$ imply $\left.Z \hookrightarrow\left[D\left(B^{m}\right)\right]\right)$.

PROOF. Let $C(t)$ be generated by $A$. We first show that for $x \in D\left(A^{m}\right)$ and $t, s \geq 0$

$$
2 C^{(2 m)}(t) C^{(2 m)}(s) x=C^{(2 m)}(t+s) x+C^{(2 m)}(|t-s|) x .
$$

In fact, set $P_{\lambda}=\lambda R\left(\lambda^{2}, A\right)$ for $\lambda>\omega$ and $y=R\left(\lambda_{0}, A\right)^{m} x$ for some $\lambda_{0}>\omega$, then

$$
\begin{array}{r}
\int_{0}^{\infty} \int_{0}^{\infty} 2 e^{-\lambda t-\mu s} C^{(2 m)}(t) C^{(2 m)}(s) y d s d t=2 P_{\lambda} P_{\mu} y=\frac{1}{\lambda-\mu}\left(P_{\mu}-P_{\lambda}\right) y+\frac{1}{\lambda+\mu}\left(P_{\lambda}+P_{\mu}\right) y \\
=\int_{0}^{\infty} \int_{0}^{\infty} e^{-\lambda t-\mu s}\left(C^{(2 m)}(t+s) y+C^{(2 m)}(|t-s|) y\right) d s d t \quad \text { for } \mu>\lambda>\omega,
\end{array}
$$

and so (8) holds for $y$. Integrating this $2 m$-times with respect to $t$ we find that the equation obtained holds for $x$. Now, (8) follows by differentiating this equation $2 m$-times with respect to $t$.

Next, let $Y$ be the completion of $X$ with respect to $\|x\|_{Y} \equiv \sup _{t \geq 0}\left\|e^{-\omega t} C^{(2 m)}(t) R\left(\lambda_{0}, A\right)^{m} x\right\|_{X}$. Then $X \hookrightarrow_{d} Y$. Since we can show by (8) and Prop.1.2 that

$$
\int_{0}^{\infty}(-s)^{k} e^{-\lambda s}\left(C^{(2 m)}(t+s) x+C^{(2 m)}(|t-s|) x\right) d s=C^{(2 m)}(t)\left(\lambda R\left(\lambda^{2}, A\right)\right)^{(k)} x
$$


for $x \in I\left(A^{m}\right), k \in \mathbf{N}_{0}$ and $\lambda>\omega$, it follows that

$$
\begin{aligned}
\left\|\left(\lambda R\left(\lambda^{2}, A\right)\right)^{(k)} \cdot x\right\|_{Y} & \leq \frac{1}{2} \sup _{t \geq 0}\left\{\int_{0}^{\infty} s^{k} e^{(\omega-\lambda) s} d s+\int_{t}^{\infty} s^{k} e^{(\omega-\lambda) s-2 \omega t} d s+\int_{0}^{t} s^{k} e^{-(\omega+\lambda) s} d s\right\}\left\|_{x}\right\|_{Y} \\
& \leq k !(\lambda-\omega)^{-k}\|x\|_{Y} \quad \text { for } \lambda>\omega, k \in \mathrm{N}_{0} \text { and } x \in X .
\end{aligned}
$$

It follows that $R\left(\lambda^{2}, A\right)$ has a unique extension $R\left(\lambda^{2}\right) \in L(Y)$ and so $R\left(\lambda^{2}\right)(\lambda>\omega)$ is a pseudo resolvent on $Y$. We show that $\lim _{\lambda \rightarrow \infty}\|\lambda R(\lambda) y-y\|_{Y}=0$ for $y \in Y$. Indeed, from (9) (with $k=0)$ it suffices to show this for $y \in X$. Let $x=R\left(\lambda_{0}, A\right)^{m} y$. Then the same method as in the proof of $[1,(3.5)]$ leads to

$$
\left\|\lambda^{2} R\left(\lambda^{2}\right) y-y\right\|_{Y}=\sup _{t \geq 0}\left\|e^{-\omega t}\left(\lambda^{2} R\left(\lambda^{2}, A\right)-1\right) C^{(2 m)}(t) x\right\|_{X} \leq I_{+}+I_{-} \rightarrow 0
$$

as $\lambda \rightarrow 0$ where $I_{ \pm}=\frac{1}{2} \sup _{t \geq 0}\left\|\int_{0}^{\infty} \lambda e^{-\lambda s}\left(C^{(2 m)}(|s \pm t|)-C^{(2 m)}(|t-s|)\right) x d s\right\|_{X}$. Thus there exists a densely defined linear oprator $B$ on $Y$ such that $\left(\omega^{2}, \infty\right) \subset \rho(B)$ and $R(\lambda)=R(\lambda, B)$ for $\lambda>\omega^{2}$. It now follows from $(9)$ that $B \in G_{0}(Y)$. The rest can be proved as in $[2, \S 3]$.

COROLLARY 5.4 If $\overline{D(A)}=X$ and $\rho(A) \neq \emptyset$. Then the following statements are equivalent.

(a) $A \in G_{2 m}(X)$.

(b) $\left[D\left(A^{m}\right)\right] \hookrightarrow Z \hookrightarrow X$ for some $Z$, and $A_{Z} \in G_{0}(Z)$.

(c) $\left[D\left(B^{m}\right)\right] \hookrightarrow X \hookrightarrow Y$ for some $Y$ and some $B \in G_{0}(Y), R\left(\lambda_{0}, B\right) X \subset X$ for some $\lambda_{0} \in \rho(B)$, and $A=B_{X}$.

After the paper was accepted, the author understood that the equivalence of (a) and (b) has been extended to the case $\overline{D(A)} \neq X$ by Shaw and $\mathrm{Li}[7]$. But Th.2.1 and 2.2 cannot be deduced by combining this with the corresponding results of cosine functions because the norm $\|\cdot\|_{m}$ is not equivalent to the norm of $Z$ (see Cor.5.4(b)).

ACKNOWLEDGEMENT. This project was supported by the National Science Foundation of China. I wish to thank Dr. deLaubenfels for sending me reference [7].

\section{REFERENCES}

1. Arendt, W. and Kiellermann, H. Integrated solutions of Volterra intergrodifferential equations and applications, Pitman Res. Notes Math. 190 (1989), 21-51.

2. Arendt, W., Neubrander, F. and Schlotterbeck, U. Interpolation of semigroups and integrated semigroups, Semigroup Forum $\underline{45}$ (1992), 26-37.

3. deLaubenfels, $R$. Integrated semigroups, $C$-semigroups and the abstract Cauchy problem, Semigroup Forum $\underline{41}$ (1990), 83-95.

4. Kellermann, H. and Hieber, M. Integrated semigroups, J. Funct. Anal. 84 (1989), 160-180.

5. Li, Y. C. and Shaw, S. Y. On generators of integrated $C$-semigroups and $C$-cosine functions, Semigroup Forum $\underline{47}$ (1993), 29-35.

6. Neubrander, F. Integrated semigroups and their applications to the abstract Cauchy problem, Pacific J. Math. 135 (1989), 111-155.

7. Shaw, S. Y. and Li, Y. C. On $n$-times integrated $C$-cosine functions, preprint, 1993.

8. Takenaka, T. and Okazawa, N. Wellposedness of abstract Cauchy problems for second order differential equations, Israel J. Math. $\underline{69}$ (1990), 257-288.

9. Travis, C. C. and Webb, G. F. Perturbation of strongly continuous cosine family generators, Colloq. Math. 45 (1981), 277-285.

10. Zheng, Q. and Lei, Y.S. Exponentially bounded $C$-semigroups and integrated semigroups with nondensely defined generators I: Approximation; Il: Perturbation (in Chinese); III: Analyticity, Acta Math. Sci. $\underline{13}$ (1993), 251-260; 13 (1993), 428-434; 14 (1994), 107-119. 


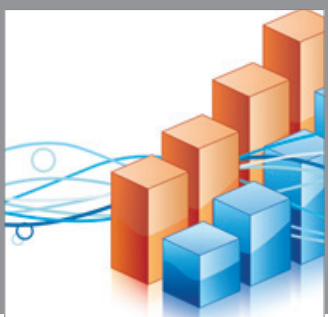

Advances in

Operations Research

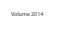

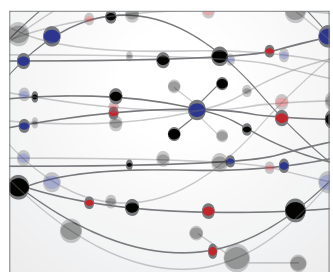

\section{The Scientific} World Journal
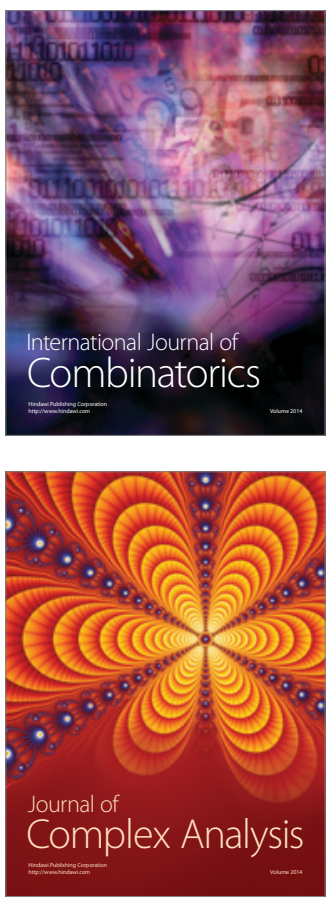

International Journal of

Mathematics and

Mathematical

Sciences
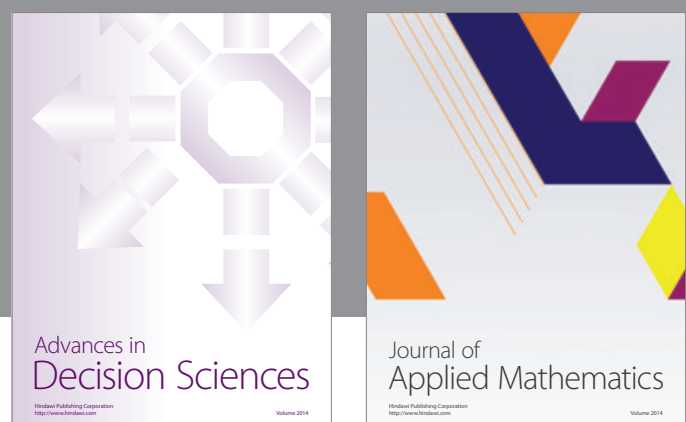

Journal of

Applied Mathematics
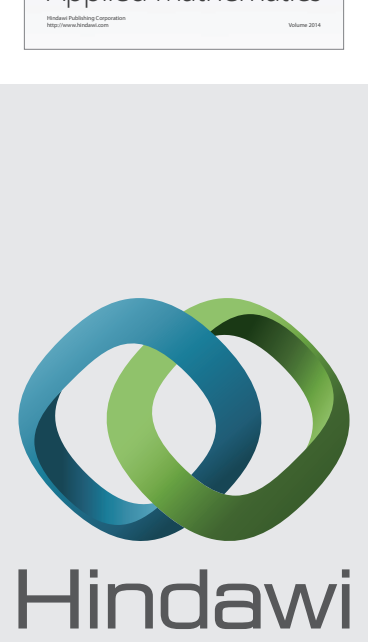

Submit your manuscripts at http://www.hindawi.com
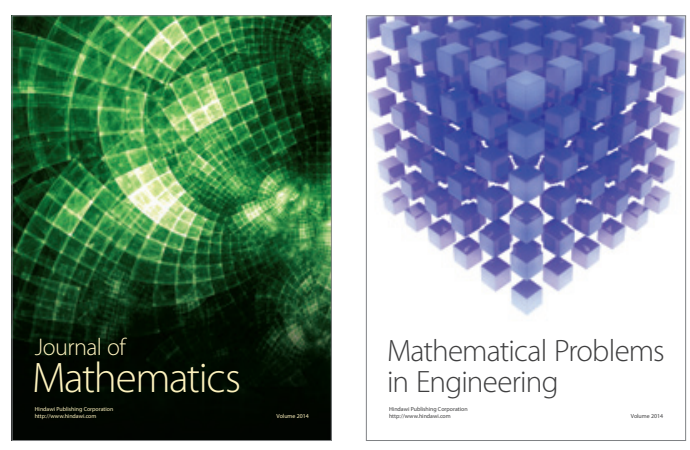

Mathematical Problems in Engineering
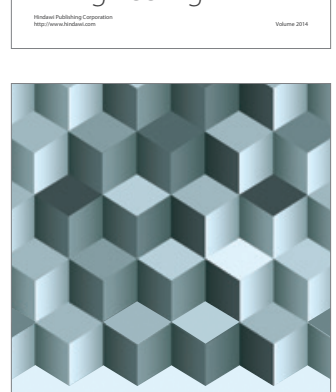

Journal of

Function Spaces
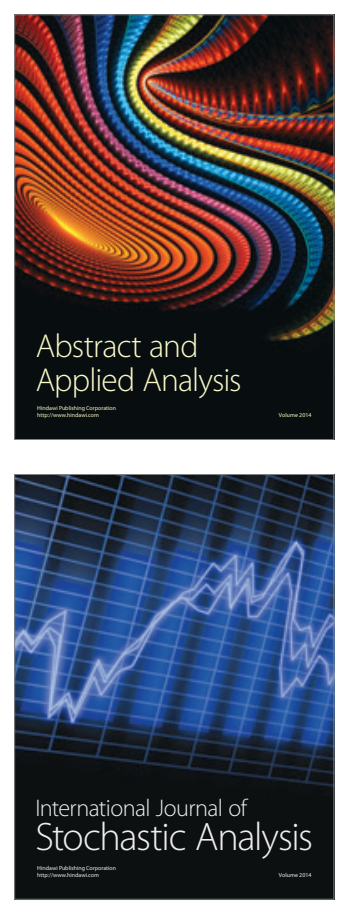

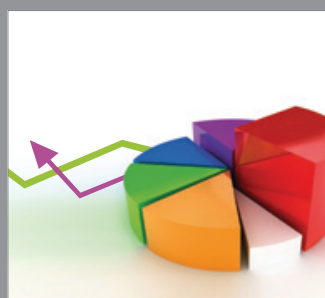

ournal of

Probability and Statistics

Promensencen
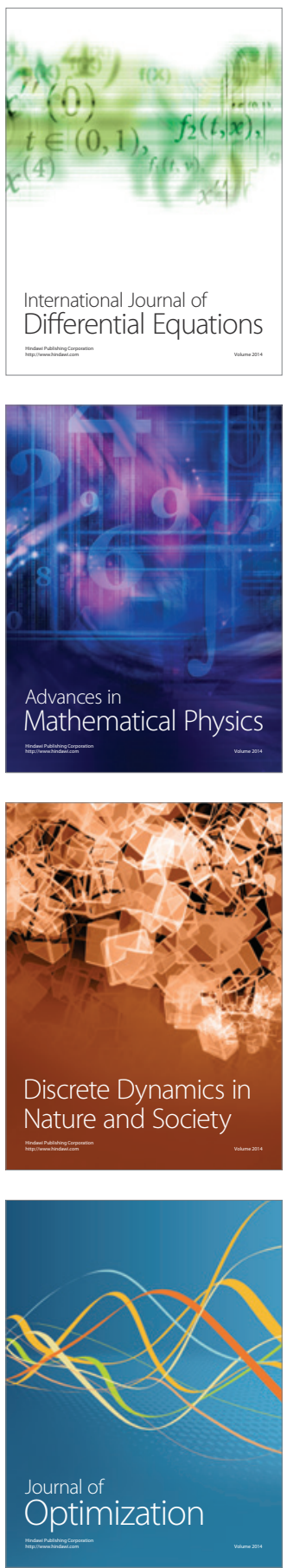